\title{
Numerical simulation of stress-strain state of oblique sections of reinforced concrete structures subjected to compression and bending on yielding supports under short-term dynamic load
}

\author{
Nikita Mescheulov ${ }^{1, *}$, and Oleg Kumpyak ${ }^{1}$ \\ ${ }^{1}$ Tomsk State University of Architecture and Building, Tomsk 634003 Russia
}

\begin{abstract}
This research is aimed at studying the method for improving blast resistance of buildings and structures by using yielding supports. The paper reports the data on dynamic analysis of reinforced concrete elements subjected to compression and bending on yielding supports under the condition of elasto-plastic stress-strain state performed in ANSYS software package. Algorithms and techniques for dynamic amplification factor calculation for reinforced concrete elements subjected to compression and bending are presented. The paper provides load diagrams of dynamic amplification factors with the account of yielding capacity of supports for subsequent dynamic analysis of structures for equivalent static load.
\end{abstract}

\section{Introduction}

Due to rapidly growing possibility of natural or man-made dynamic actions occurrence in buildings and structures, the necessity to design reinforced concrete structures resistant to such actions is becoming ever more frequent. Special attention is paid to the risk of shock waves caused by explosions of conventional explosives during their storage, transportation, or as a result of terrorist attacks, etc.

Because of short period of action and high intensity loads that occur in such cases often result not only in local damage or complete failure of a structure, but also cause loses of technological equipment, injuries and casualties. For that reason solution to the issue of blast resistant buildings and structures has an important economic and social meaning.

Presently, two essentially different types of solution are used to ensure accommodation of dynamic loads by buildings and structures.

The first type provides increase of bearing capacity of the structures by using materials with improved physico-mechanical characteristics (high-performance concrete and rebars, composite rebars, fiber concrete), more intense reinforcement, increase of cross-section of elements. At the same time, structures performance in regions of massive plastic deformations is acceptable.

\footnotetext{
* Corresponding author: nikita.mesheulov@mail.ru
} 
Bended elements and structures under compression and bending are the main ones when analyzing buildings and structures for highly intense dynamic actions. Such structures include floor slabs, cross beams, columns. Occurrence of high plastic deformations may result in local or complete failure of a building. Besides, enhancing dynamic strength by improving geometrical and strength parameters of structures is not always technologically possible or economically feasible.

An alternative protection option is decrease of intensity and containment of dynamic action by using both blast relief elements (floor slabs and envelope structures) and brittle elements and devices. In the first case, the building design provides failure of support fixing of some elements under the action of shock waves, which causes detachment of load bearing structures and release of shock waves through such openings. In the second case, it is suggested to install in buildings some structures or devices (yielding supports [1-8]) which when deforming will take up excessive blast energy, thus unloading and protecting bearing elements from damage and failure.

Today, the research outcomes in the field of using yielding supports for protection of structures damaged by extremely intense dynamic loads are only partially presented. The reported experimental and theoretical studies [9-13] demonstrate both positive and negative impact of yielding capacity of support fixing on dynamic response of reinforced concrete (RC) bended structures.

Hence, the research aimed at studying RC structures subjected to bending and compression with yielding support fixing under short-term dynamic loading is relevant and has important meaning while designing economically effective and reliable buildings and structures.

\section{Calculation method}

Simulation of dynamic deformation of oblique sections of RC bended structures and elements subjected to bending and compression on rigid and yielding supports was carried out using Ansys Explicit Dynamics software, version 17.2.

Under study is a beam subjected to compression and bending in conventional elastic stage corresponding to limit state $1^{\text {a }}$ [14]. The level of longitudinal compression is varied, as well as two stages of supports deformation: conventional non-deformable (rigid) stage and elasto-plastic stage [10].

The calculation is performed for a RC beam structure subjected to bending and compression with the section parameters of $h=40 \mathrm{~cm}, b=20 \mathrm{~cm}$, and with the span of $l=4 \mathrm{~m}$. In normal sections the beam is reinforced with hot-rolled A400 $\left(\mu_{s}=2 \%\right)$ rebars. In oblique sections the beam is reinforced with hot-rolled A240 rebars. Bilinear dependence of plastic deformation of steel with isotropic hardening was used in the calculation as a law of longitudinal and transverse reinforcement deformation. Concrete of B20 class was used and described with Riedel-Hiermaier-Thoma (RHT) model of combined plasticity and failure. The beam is designed with equal strength for normal and oblique sections.

Finite element (FE) model (Fig. 1) consists of a RC element 1, moveable 2 and immoveable 3 support plates and a yielding support 4 . The research focuses on one quarter of the initial structure due to its axisymmetric geometry. 


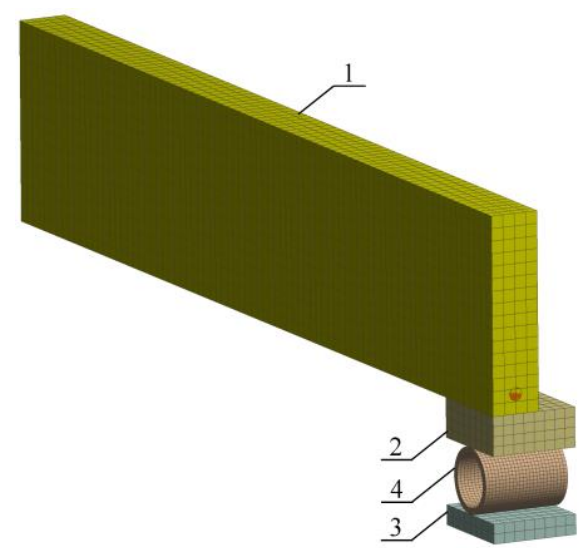

Fig. 1. General view of the FE model: 1 - reinforced concrete sample; 2 - moveable support plate; 3 - immoveable loading support plate; 4 - yielding support

For this reason the rest three quarters of the model were replaced by horizontal perpendicular symmetry planes (Fig. 2) describing the reverse behavior of the sample parts.

a)

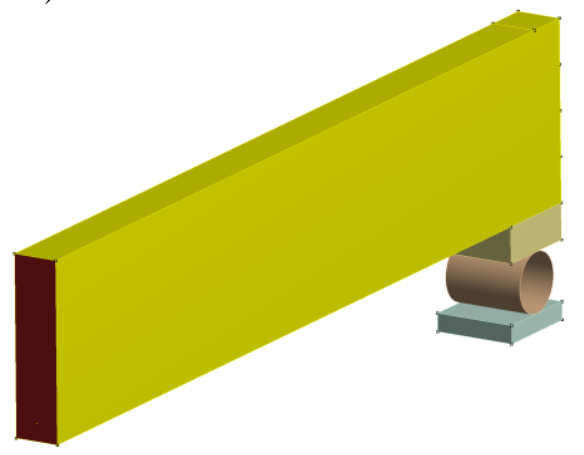

b)

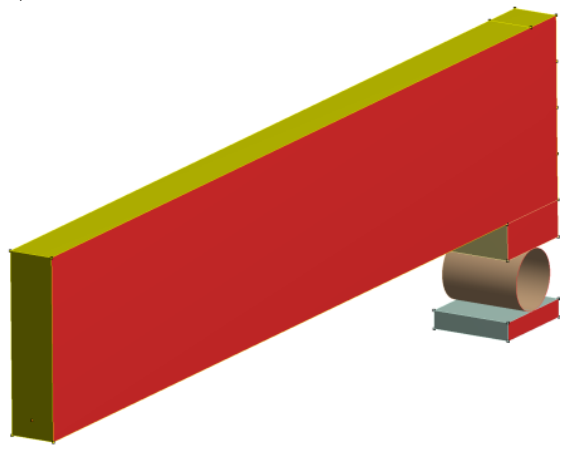

Fig. 2. Symmetry planes (in red): $a$ - transverse; $b$ - longitudinal

In cases of calculation of structures subjected to bending and compression the first stage provided application and retention of longitudinal force (Fig. 3a) which is applied as evenly distributed load along the entire side surface of the beam and equals $(0 ; 0.2 ; 0.4) R_{b n}$, where $R_{b n}$ is characteristic compressive strength of concrete (MPa).

a)

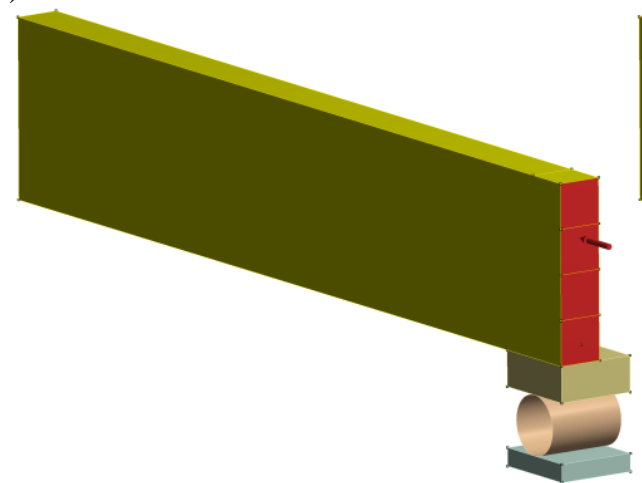

b)

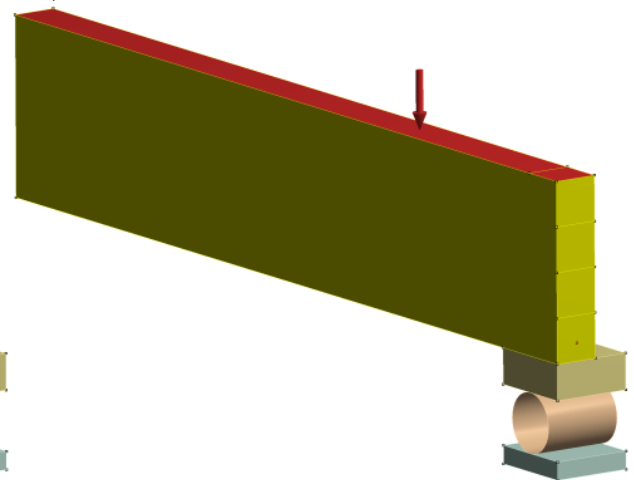

Fig. 3. Load application: a - longitudinal static load; b - transverse dynamic load 
After that, simulation of transverse dynamic action (Fig. 3b) was performed with regard to immediately increased load with linear descent (Fig. 4).

This linear manner of dynamic load variation in time is applied for calculation of roof structures, side and front load bearing structures in buildings during external explosion of condensed explosives [15].

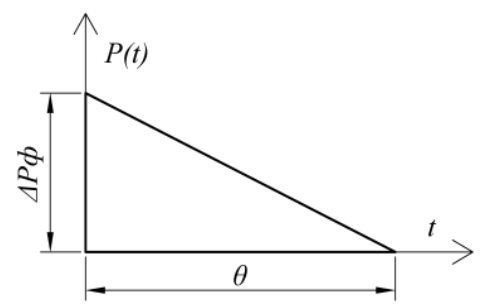

Fig. 4. Immediate increase of dynamic load with linear descent

When calculating the linearized law of pressure variation in time was applied which is defined by the dependence (1).

$$
\Delta P_{(t)}=\Delta P_{\max }\left(1-\frac{t}{\theta}\right), 0 \leq t \leq \theta
$$

The shock wave effective time $(\theta)$ was set depending on multiple parameters: distance from the charge center, mass of the charge, distance between the charge and the affected building. In calculation of serviceability limit state only conventional elastic stage of structural deformation (limit state $1^{\text {a }}$ ) is provided. In RC structures with longitudinal reinforcement deformations of tensed rebars stay within elastic deformation stage, however in tension zones crack formation is possible. Following the recommendations in [15], the time of elastic stage end $t_{e 2}$ when calculating according to the limit state $1^{\text {a }}$ is defined by dependences (2) and (3)(3).

where,

$$
\begin{aligned}
& t_{e 2}=\left(\frac{2 \operatorname{arctg} \omega_{1} \theta}{\omega}\right), \text { with } \omega_{1} \theta \geq 2.33 \\
& \operatorname{tg} \omega_{1}\left(t_{e 2}-\theta\right)=\frac{A}{B}, \text { with } \omega_{1} \theta<2.33
\end{aligned}
$$

$$
\begin{gathered}
A=-\frac{1}{\omega_{1} \theta}+\sin \omega_{1} \theta+\frac{\cos \omega_{1} \theta}{\omega_{1} \theta} \\
B=-\cos \omega_{1} \theta+\frac{\sin \omega_{1} \theta}{\omega_{1} \theta}
\end{gathered}
$$

First mode natural frequencies $\omega_{1}$ are calculated on the basis of the set geometrical and physical parameters. After that, the parameter $\theta$ is defined from the dependences (2) and (3) for a certain calculation case.

The maximum value of dynamic load $\left(\Delta P_{\max }\right)$ is determined with the method of successive approximations by using iterative structural analysis with controlling occurrence of limit states $1^{\mathrm{a}}$ or $1^{\mathrm{b}}$ based on deformation criterion.

In calculation referring to load bearing capacity (or plastic deformation limitation) the state $1^{\mathrm{b}}$ is the time of the end of elastic stage $t_{e l}$, we accept $t_{e l}=0.5 t_{e 2}$. Natural frequency of a simply supported beam without longitudinal compression is determined from the dependence (6)

$$
\omega_{1}=\frac{\pi^{2}}{l^{2}} \sqrt{\frac{B}{m}}
$$


where $m$ - mass per unit length expressed in $\mathrm{t} / \mathrm{m}, B$ - bending stiffness of RC elements with rectangular section with a single rebar and cracks in tension zone of concrete, expressed in $\mathrm{kN} / \mathrm{m}^{2}$. This parameter is defined for standard static material resistance with the account of cracks in concrete of tension zone and is found from the formula (7) .

$$
B=0.8 E_{s} A_{s}\left(h_{0}-x\right)\left(h_{0}-0.5 x\right)
$$

where $E_{s}$ - rebar elastic modulus, $A_{s}$ - area of cross-section of tensed rebar; $h_{0}$ - effective depth of section, $x$ - height of compression zone of concrete.

After finding $\Delta P_{\max }$ dynamic amplification factor $k_{d}=P_{v} / \Delta P_{\max }$ is set for certain values of $\omega_{1} \theta$.

\section{Results}

\subsection{Beam structures with longitudinal compression on rigid supports}

The diagram (Fig. 5) illustrates the obtained results for calculating dependence of dynamic amplification factor for beams with and without longitudinal compression in conventional elastic stage. The data are given for a certain limit state $1^{\mathrm{a}}$ and rigid support depending on the product of first mode natural frequencies $\left(\omega_{1}\right)$ and time of dynamic load application $(\theta)$.

Good convergence was found between the research outcomes of this study and other previously reported results (Fig. 5) on the use of dynamic amplification factor $k_{d}$ for structures on rigid supports $[14,15]$ and elastic supports with higher stiffness [13]. The difference is between $0 \%$ and $6 \%$ depending on $\omega_{1} \theta$ parameter.

Reduction of dynamic amplification factor of the load $k_{d}$ is observed for different levels of longitudinal compression (Fig. 5, 4; 5). For instance, the average reduction for beams subjected to longitudinal compression $N=0,2 N_{\max }$ compared to beams without compression is 1.52 times.

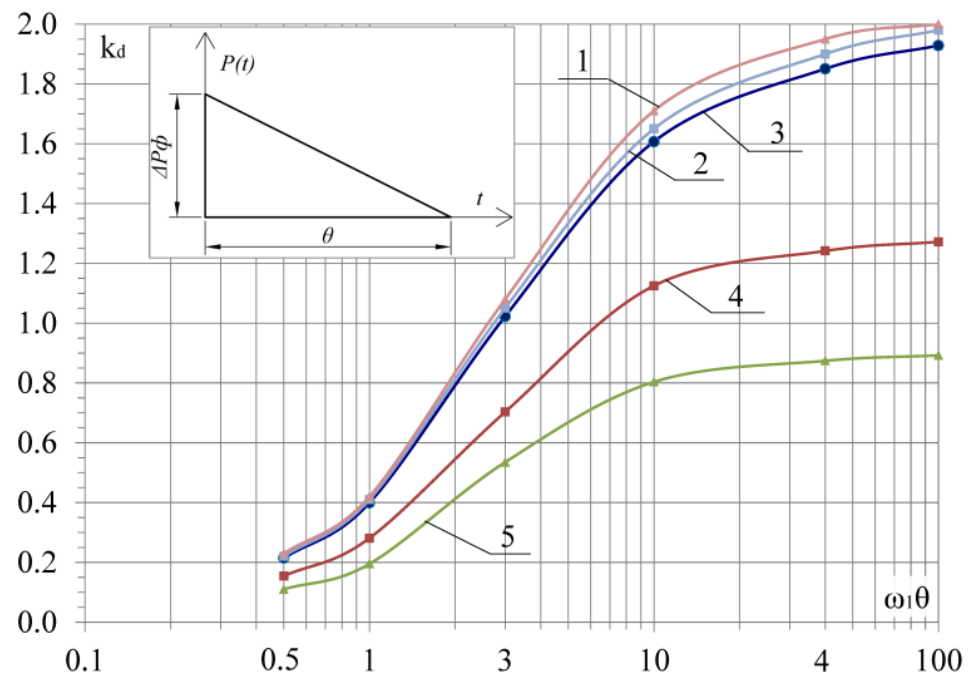

Fig. 5. Diagram for dynamic amplification factor for beams in conventional elastic stage (limit state $1^{\mathrm{a}}$ ) on rigid supports depending on the level of longitudinal compression and $\omega_{1} \theta$ parameter; 1 - B.S. Rastorguev, A.I. Plotnikov, D.Z. Khusnutdinov [15] (no longitudinal compression); 2 - A.S. AbdulRakhman [13] (no longitudinal compression); 3, 4, 5- the authors' research outcomes, 3 - beams without compression, 4 - structures subjected to longitudinal compression $N=0,2 N_{\max }, 5$ - beams subjected to longitudinal compression $N=0,4 N_{\max }$ 
When increasing compression up to $N=0,4 N_{\max }$ reduction of $k_{d}$ in comparison with beams under compression $N=0,2 N_{\max }$ is 1.4 times, whereas compared to beams without compression $k_{d}$ parameter reduces by 2 to 2.2 times.

It should be noted that increase of longitudinal compression leads to reduction of dynamic amplification factor irrespective of $\omega_{1} \theta$ parameter. For instance, for $\omega_{1} \theta=0.5$ reduction of $k_{d}$ factor for beams subjected to compression $N=0,4 N_{\max }$ is 2.09 times compared to that for beams with no compression; and for $\omega_{1} \theta=100$ the factor reduction is 2.17 times. At the same time, increase of dynamic amplification factor $k_{d}$ was observed along with growth of $\omega_{1} \theta$ parameter. Thus, for a structure with no compression and $\omega_{1} \theta=$ 0.5 the value of dynamic amplification factor $k_{d}$ is 0.23 , whereas for $\omega_{1}=100$ is equals 1.93. For beam structures subjected to bending without compression the value of $k_{d}$ decreases by 8.8 times, for beams subjected to compression $N=0,2 N_{\max }-$ by 8.5 times, and for structures subjected to compression $N=0,4 N_{\max }-$ by 8.1 times.

\subsection{Beam structures on yielding supports under various level of compression}

The dynamic factor diagram (Fig. 6) demonstrates dependences of $k_{d 2}$ factors for beams on supports in elasto-plastic stage.

When increasing compression up to $N=0,4 N_{\max }$ the $k_{d 2}$ factor reduces by 1.5 to 2 times compared to the case of the beams subjected to compression $N=0,2 N_{\max }$. With reference to beams with no compression the $k_{d 2}$ factor reduces by 2.6 to 2 times as $\omega_{1} \theta$ parameter increases.

The analysis of performance of beams on yielding supports in comparison with that on rigid supports with the account of longitudinal compression showed that the least desired combination is deformation of structures on rigid supports without longitudinal compression (Fig. 5, 1-3).

Under these conditions the values of dynamic amplification factor $k_{d}$ varies between 0.22 and 1.93 depending on $\omega_{l} \theta$ parameter. The higher $\omega_{l} \theta$ value is, the higher $k_{d}$ factor is. Performance of beams subjected to longitudinal compression $N=0,4 N_{\max }$ on yielding supports deformed in elasto-plastic stage is found to be the most effective (Fig. 6, 3). For such combination $k_{d 2}$ value belongs to the range between 0.03 and 0.46 .

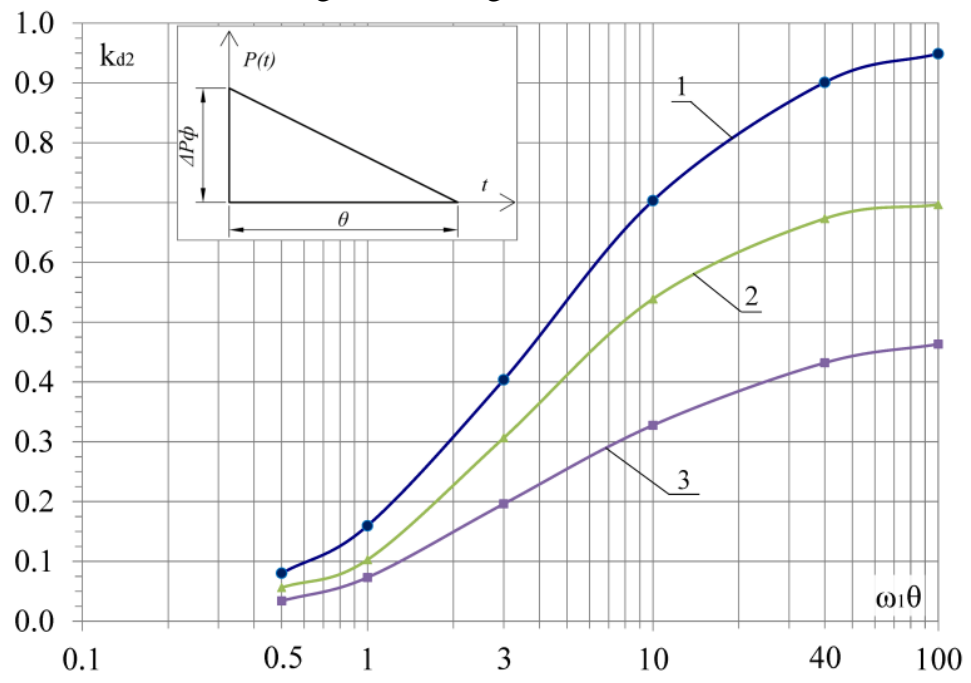

Fig. 6. Dynamic factor diagram for beams in conventional elastic stage (limit state 1a) on yielding supports subjected to various compression depending on $\omega_{1} \theta$ parameter, 1 - no compression, 2 compression $N=0,2 N_{\max }, 3$ - compression $N=0,4 N_{\max }$ 
Notably, similar to the case of rigid supports the increase of $k_{d 2}$ factor occurs along with the increase of $\omega_{1} \theta$ value. Reduction of $k_{d 2}$ factor value varies between 6.3 and 4.2 times. The best result corresponds to the lowest value of $\omega_{1} \theta$. And with increase of this parameter the mentioned effect decreases. Thus, with $\omega_{1} \theta=0.5$ for a beam with no compression on rigid supports the factor $k_{d}=0.21$, and for a beam on yielding supports under compression $N=0,4 N_{\max }$ the factor $k_{d 2}=0.03$. This means that the factor reduced by 6.3 times. With $\omega_{1} \theta$ $=10$, for a beam without compression on rigid supports the value of $k_{d}$ factor is 1.61 , whereas for structures on yielding supports under compression $N=0,4 N_{\max }$ it is 0.33 . The factor reduction in this case is 4.9 times. With $\omega_{l} \theta=10$, taking a beam with no compression on rigid supports the factor $k_{d}$ value is 1.93 , and for a beam on yielding supports and subjected to compression $N=0,4 N_{\max }$ the $k_{d 2}$ value is 0.46 . Hence, the factor reduction is 4.2 times.

Comparative analysis of dynamic resistance of RC structures demonstrates that longitudinal compression and yielding supports have significant effect on bearing capacity of the element. Notably, supports that perform in plastic stage prove to be the most effective.

\section{Conclusion}

1. Forces occurring in longitudinal rebars and corresponding to the limit state 1a are characterized by dynamic amplification factors $k_{d}, k_{d 2}$. The obtained factors are presented in the form of diagrams, which significantly simplifies analysis of reinforced concrete beam structures for equivalent static loads.

2. Dynamic amplification factors $k_{d}, k_{d 2}$ depend on the following parameters: relation of yielding support stiffness and structure stiffness $(W)$, product of first mode natural frequencies $\left(\omega_{1}\right)$ and time of dynamic load application $(\theta)$, and the level of longitudinal compression.

3. Reduction of dynamic amplification factors is observed for structures deformed in conventional elastic stage with the increase of longitudinal compression up to $N=0,4 N_{\max }$. In case of rigid supports such reduction is up to 2 times, and for yielding supports - up to 2.6 times.

4. Relation of yielding support stiffness and structure stiffness is an essential parameter for dynamic amplification factors $k_{d 2}$. Decrease of its value for yielding support causes reduction of $k_{d 2}$ up to $90 \%$.

5. Performance of a beam subjected to longitudinal compression $N=0,4 N_{\max }$ on yielding supports in plastic stage proves to be the most effective. Thus, reduction of dynamic amplification factor $k_{d 2}$ for beams on yielding supports compared to the dynamic amplification factor $k_{d}$ for rigid supports reaches between 4.2 and 6.3 times.

\section{References}

1. S. Poonaya, C. Thinvongpituk and U. Teeboonma, Proceedings of WASET, 26, 329 334 (2007)

2. P. Mata, A. Barbat, S. Oller and R. Boroschek, Arch. comput. methods eng, 15, 489 539 (2008)

3. G. Nagel, "Impact and energy absorption of straight and tapered rectangular tubes", Ph.D. thesis, Queensland University of Technology (2005)

4. A. Olabi, E. Morris and M, Hashmi, Journal of Thin-Walled Structures, 45, 706-726 (2008) 
5. A. Ahmad Zaidi and G. Ling Lang, Journal of Science and Technology, 2, 563 - 580 (2011)

6. J. Marzbanrad, M. Mehdikhanlo and A. Saeedi Pour, Turkish J. Eng. Env., 33, 159 166 (2009)

7. Y. Zhao and J. Fang, International Journal of Pressure Vessels and Piping, 67, 257 261 (1996)

8. A. Ghaidan, Tikrit Journal of Eng. Sciences, 18 (3), 52-60 (2011)

9. O. Kumpyak, Z. Galyautdinov and D. Kokorin, II All-Russian Scientific Conference of Young Scientists “Advanced Materials in Technology and Construction”, 1698, (2015) (in Russian)

10. O. Kumpyak and N. Meshcheulov, Vestnik TSUAB, 6, 70-80 (2014) (in Russian)

11. A. Kezmane, B. Chiaia, O. Kumpyak, V. Maksimov and L. Placidi, European Journal of Environmental and Civil Engineering, 20, 1-38 (2016)

12. B. Rastorguev, Building mechanics and analysis of structures, 1, 50-57 (2009) (in Russian)

13. A.S. Abdul-Rakhman, Increase of load-bearing capacity of concrete structures under impact loads, (PhD Thesis, Moscow, 1995) (in Russian)

14. SP 88.13330.2014 The protective shelters of civil defense, 122, (2013). (in Russian)

15. B.S. Rastorguev, A.I. Plotnikov, D.Z. Khusnutdinov, Proektirovanie zdaniy $i$ sooruzheniy pri avariynykh vzryvnykh vozdeystviyakh, 15, 2, (2007) (in Russian) 